\title{
Noninvasive follicular thyroid neoplasm with papillary-like nuclear features
}

\author{
Pedro Weslley Rosario, Gabriela Franco Mourão, Maurício Buzelin Nunes, \\ Marcelo Saldanha Nunes and Maria Regina Calsolari
}

Santa Casa de Belo Horizonte, Minas Gerais, Brazil

Correspondence should be addressed to P W Rosario Email pedrowsrosario@gmail.com

\begin{abstract}
Recently, it was proposed that some papillary thyroid carcinomas (PTC) will no longer be termed 'cancer' and are christened as 'noninvasive follicular thyroid neoplasm with papillary-like nuclear features' (NIFTP). As this is a recent definition, little information is available about NIFTP. The objective of this study was to report the frequency, ultrasonographic appearance, cytology result and long-term evolution of cases of NIFTP seen at our institution. We excluded tumours $\leq 1 \mathrm{~cm}$. The sample consisted of 129 patients. Sixty-four patients were submitted to total thyroidectomy and 65 to lobectomy. These patients with NIFTP did not receive radioiodine. NIFTP corresponded to $15 \%$ of cases diagnosed as PTC $>1 \mathrm{~cm}$. An ultrasonographic appearance considered to be of low suspicion for malignancy was common in NIFTP $(32.5 \%)$, whereas a highly suspicious appearance was uncommon (5\%). NIFTP frequently exhibited indeterminate cytology $(62 \%)$, while malignant cytology was uncommon (4\%). The patients were followed up for 12-146 months (median 72 months) after surgery. None of the patients developed structural disease during follow-up. Comparing the concentrations of thyroglobulin ( $\mathrm{Tg}$ ) and anti-Tg antibodies ( $\mathrm{TgAb}$ ) obtained 6-12 months after surgery and in the last assessment, none of the patients exhibited an increase in these markers.
\end{abstract}

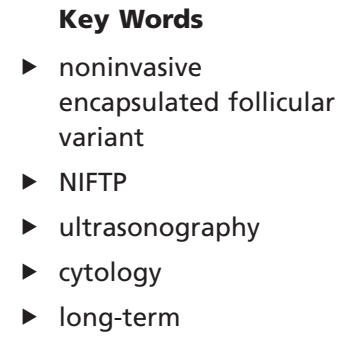

Endocrine-Related Cancer (2016) 23, 893-897

\section{Introduction}

Recently, it was proposed that some papillary thyroid carcinomas (PTC) will no longer be termed 'cancer' and instead be called 'noninvasive follicular thyroid neoplasm with papillary-like nuclear features' (NIFTP) (Nikiforov et al. 2016). This tumour has so far been diagnosed as 'noninvasive encapsulated follicular variant of PTC' (noninvasive E-FVPTC). Recurrence was not observed in patients with NIFTP (Nikiforov et al. 2016) and was very rare in patients with noninvasive E-FVPTC of previous series, even when they were submitted only to lobectomy (Nikiforov et al. 2016). As most physicians still manage these cases similar to classical PTC, the name change aims to reduce overtreatment and to minimize the psychological impact of the diagnosis of 'cancer' (Nikiforov et al. 2016). It is estimated that about one-fifth of patients currently diagnosed with PTC could have their diagnosis changed to NIFTP (Nikiforov et al. 2016).

It is important to point out that not all cases diagnosed previously as noninvasive E-FVPTC can automatically be considered NIFTP, as the diagnostic criteria for E-FVPTC were not uniform and well-defined criteria for NIFTP were proposed only now (Nikiforov et al. 2016). As the 


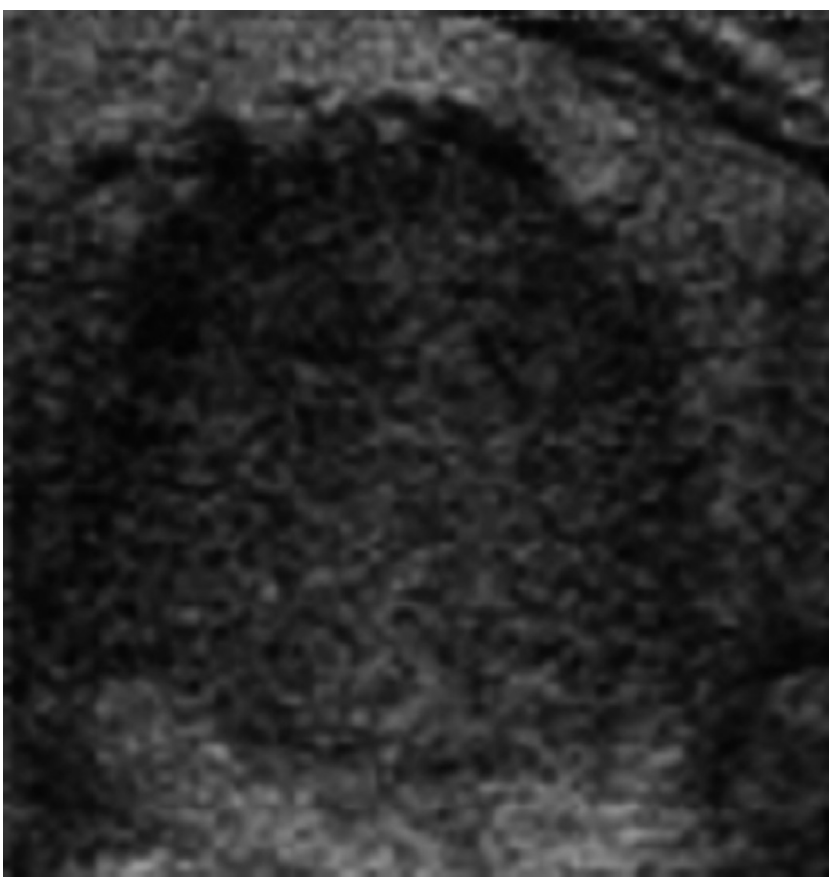

Figure 1

Hypoechoic nodule that is taller than wide (high suspicion).

definition is recent, very little information is available about NIFTP. Most studies describe the preoperative characteristics of FVPTC in general without a distinction between the encapsulated and non-encapsulated form. Few studies report the characteristics of E-FVPTC but group tumours with and without invasion/infiltration, and specific information about noninvasive E-FVPTC is scarce (Jang et al. 2015). As we can see, not all cases diagnosed previously as noninvasive E-FVPTC meet the careful definition of NIFTP (Nikiforov et al. 2016). Furthermore, on account of the removal of the designation 'cancer' will imply, as desired, a reduction in management (treatment and follow-up), more data regarding the long-term evolution of these tumours are desirable.

We published previously the evolution of 57 patients with noninvasive E-FVPTC $>1 \mathrm{~cm}$ submitted only to lobectomy (Rosario et al. 2014). The objective of this study was to report the frequency, ultrasonographic appearance, cytology result and long-term evolution of cases of NIFPT (defined using the recent criteria of Nikiforov et al. (2016)) seen at our institution.

\section{Patients and methods}

This was a retrospective study. First, we reviewed the cases of all patients with a diagnosis of noninvasive E-FVPTC seen at our institution: (i) thick, thin or partial

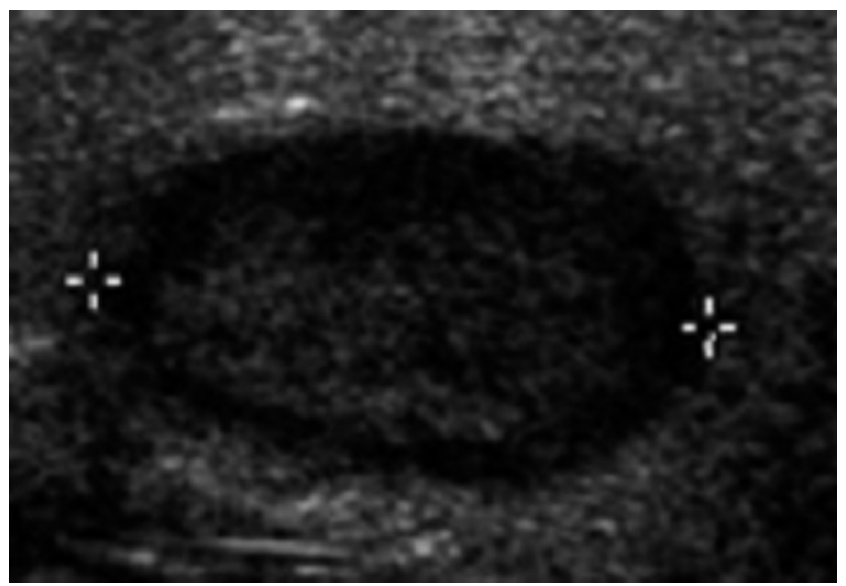

Figure 2

Hypoechoic nodule without high suspicion features (intermediate suspicion).

capsule or well circumscribed with a clear demarcation from adjacent thyroid tissue; (ii) follicular growth pattern; (iii) unequivocal nuclear changes of PTC; and (iv) absence of capsular or vascular invasion. Tumours with insufficient nuclear changes for the diagnosis of PTC did not receive the diagnosis of E-FVPTC as they do not meet criterion (iii) described above. Finally, to meet the definition of NIFTP (Nikiforov et al. 2016), 8 cases were excluded because they exhibited 'true' papillae $>1 \%$ or cell/morphologic characteristics of other variants of PTC. We also excluded tumours $\leq 1 \mathrm{~cm}(n=5)$. The final sample consisted of 129 patients.

The results of preoperative ultrasonography (US) and cytology were reviewed. Based on the US features, the nodules corresponding to the tumour (NIFTP) were classified according to ATA (Haugen et al. 2016) as high suspicion (Fig. 1), intermediate suspicion (Fig. 2), low suspicion (Fig. 3), very low suspicion or undefined. The last classification corresponded to iso- or hyperechoic nodules with some suspicious finding, that is, irregular margins, microcalcifications, taller than wide shape or rim calcifications with a small extrusive soft tissue component (Haugen et al. 2016). The results of cytology were classified according to the Bethesda System as (i) non-diagnostic, (ii) benign, (iii) follicular lesion or atypia of undetermined significance, (iv) suspicious for or follicular neoplasm, (v) suspicious for malignancy and (vi) malignant (Haugen et al. 2016).

Sixty-four patients were submitted to total thyroidectomy and 65 to lobectomy. Radioiodine was not administered to any of the 129 patients with NIFTP. Seven patients had papillary microcarcinoma associated with the main tumour.

Published by Bioscientifica Ltd 


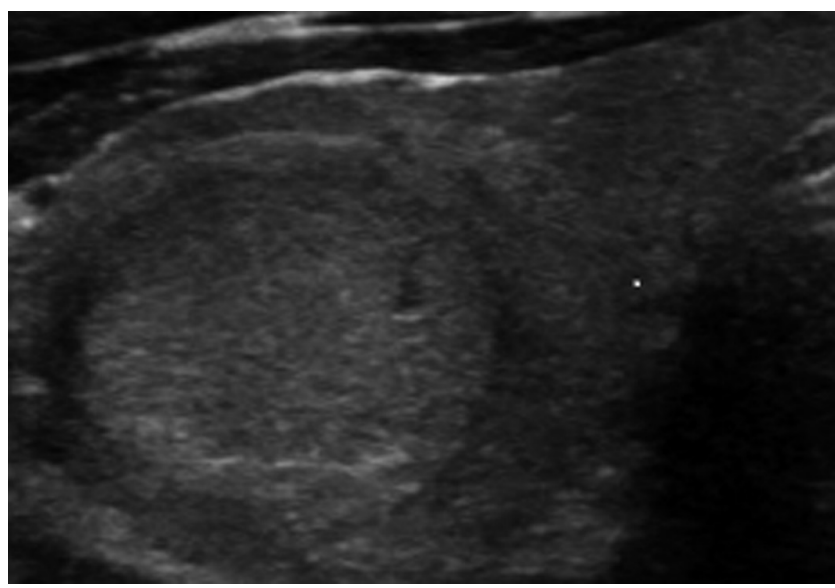

Figure 3

Isoechoic nodule without high suspicion features (low suspicion).

After surgery, levothyroxine therapy was initiated to maintain TSH between 0.5 and $2 \mathrm{mIU} / \mathrm{L}$. Approximately 6 months later, the patients were evaluated by clinical examination, chest X-ray, neck US, and measurement of thyroglobulin (Tg) and anti-Tg antibodies (TgAb). Subsequently, clinical examination, unstimulated $\mathrm{Tg}$, $\mathrm{TgAb}$ and US were obtained annually.

\section{Results}

There were 110 women and 19 men ranging in age from 16 to 76 years (mean 46 years). Median tumour size was $3.5 \mathrm{~cm}$ (range, 1.1-7). Among the cases diagnosed at our institution as PTC $>1 \mathrm{~cm}, 15 \%$ were NIFTP.

An US appearance defined as low suspicion of malignancy by ATA (Haugen et al. 2016) was common in NIFTP, while a highly suspicious appearance was rarely observed (Table 1). NIFTP more frequently exhibited indeterminate cytology (Bethesda categories iii or iv) $(62 \%)$, while malignant cytology was uncommon $(4 \%)$ (Table 2).

Lymph nodes were removed in 12 patients ( 2 to 11 lymph nodes), but lymph node metastases were confirmed in only one case that had a papillary microcarcinoma associated with NIFTP. Complete resection of the primary tumour was achieved in all patients.

The patients were followed up for 12-146 months (median 72 months). None of the patients developed structural disease during follow-up after surgery. Table 3 shows the behaviour of $\mathrm{Tg}$ and $\mathrm{TgAb}$ comparing the concentrations obtained 6-12 months after surgery and in the last assessment.

\section{Discussion}

First, we emphasize that this study included a large number of patients with NIFTP $(n=129)$ using the recommended criteria (Nikiforov et al. 2016). To our knowledge, this is the first study to describe the US appearance of these lesions and the second that has reported the results of cytology (Maletta et al. 2016). We also report the evolution after a follow-up period of up to 12 months for patients not treated with radioiodine, half of them submitted only to lobectomy. In contrast to the multicentre study (Nikiforov et al. 2016), which included little information about the follow-up protocol of the patients (Pradeep 2016), this protocol was uniform in this study and followed that recommended for patients not submitted to radioiodine (Haugen et al. 2016), including periodic neck US. In addition, the behaviour of $\mathrm{Tg}$ and $\mathrm{TgAb}$ in the last assessment was reported.

As in the original publication (Nikiforov et al. 2016), some cases diagnosed previously as noninvasive E-FVPTC did not meet the definition of NIFTP, which is more rigorous. Nevertheless, at our institution NIFTP corresponded to $15 \%$ of the patients diagnosed with PTC, showing the relevance of these lesions in clinical practice and the negative impact if these patients were overtreated (surgical complementation, radioiodine, TSH suppression) and submitted to more rigorous follow-up.

Using the US classification of ATA (Haugen et al. 2016 ), in the case of nodules $>1 \mathrm{~cm}, 63.3 \%$ of lesions

Table 1 Result of ultrasonography in NIFTP.

\section{Category according to the ultrasonographic classification of the} American Thyroid Association (Haugen et al. 2016)

\section{Undefined}

Very low suspicion

Low suspicion

Intermediate suspicion

High suspicion

\begin{tabular}{c}
\hline NIFTP $(n=120) *$ \\
$5(4.1 \%)$ \\
0 \\
$39(32.5 \%)$ \\
$70(58.3 \%)$ \\
$6(5 \%)$ \\
\hline
\end{tabular}

NIFTP, noninvasive follicular thyroid neoplasm with papillary-like nuclear features. *Ultrasonography was not available for 9 patients. 
Table 2 Result of cytology in NIFTP.

\begin{tabular}{lcc}
\hline Bethesda category & & NIFTP $(n=126)^{*}$ \\
\cline { 1 - 2 } I (non-diagnostic) & $1(0.8 \%)$ \\
II (benign) & $10(8 \%)$ \\
III (AUS or FLUS) & $25(20 \%)$ \\
IV (suspicious for NF or NF) & $53(42 \%)$ \\
V (suspicious for malignancy) & $32(25.4 \%)$ \\
VI (malignant) & $5(4 \%)$ \\
\hline
\end{tabular}

AUS, atypia of undetermined significance; FLUS, follicular lesion of undetermined significance; NF, follicular neoplasm; NIFTP, noninvasive follicular thyroid neoplasm with papillary-like nuclear features.

*Cytology was not available for 3 patients.

measuring up to $1.5 \mathrm{~cm}$ and $95 \%$ of lesions $>1.5 \mathrm{~cm}$ would have an indication for fine-needle aspiration (FNA). Thus, according to the current indications (Haugen et al. 2016), most of these lesions (NIFTP) will be submitted to FNA. In contrast to classic PTC that more frequently exhibits a highly suspicious appearance on US and rarely a low suspicion (Jang et al. 2015; Rosario PW \& Silva AL, unpublished observations), a highly suspicious appearance was uncommon in NIFTP (5\%) and one-third of the patients had US of low suspicion. To our knowledge, no study has so far reported this information (US in NIFTP), but a previous series analyzed the characteristics of noninvasive E-FVPTC $(n=20)$ and also found that highly suspicious nodules on US are uncommon (Jang et al. 2015).

Regarding cytology, approximately $25 \%$ of the cases of NIFTP exhibited cytology suspicious for malignancy (PTC). This percentage is similar to that reported in a previous study that specifically evaluated the cytology result in NIFTP (Maletta et al. 2016). In addition, in noninvasive E-FVPTC, $25-45 \%$ of cases can have cytology suspicious for PTC (Strickland et al. 2015, Faquin et al. 2016, Mehrzad et al. 2016). In these cases, it has been proposed that a predominance of microfollicles without papillae, pseudoinclusions or psammomatous calcifications would identify lesions that are more likely to be NIFTP (Strickland et al. 2016). Cytology was indeterminate in approximately two-third of cases of NIFTP in this study and in another series (Maletta et al. 2016), and in 30-70\% of cases of noninvasive E-FVPTC in previous studies (Strickland et al. 2015, Faquin et al. 2016, Mehrzad et al. 2016). When the nuclear alterations required for the histological diagnostic of NIFTP (score 2 or 3 (Nikiforov et al. 2016)) are observed in cytology classified as indeterminate, benign follicular lesions are unlikely, but the distinction between NIFTP and invasive E-FVPTC is not possible (Maletta et al. 2016).
Table 3 Behaviour of $\mathrm{Tg}$ and $\mathrm{TgAb}$ (6-12 months after surgery vs last assessment) in patients with NIFTP.

\begin{tabular}{lcc}
\hline Patients initially without TgAb $(n=108)$ & $\boldsymbol{N}(\%)$ \\
\hline Tg increase & & 0 \\
Positive TgAb & & $22(20.4)$ \\
Stable Tg & & \\
Tg reduction $>20 \%$ & $(79.6)$ \\
Patients initially with positive TgAb & \\
$(n=21)^{\star}$ & \\
TgAb increase & $5(23.8)$ \\
Stable TgAb & $6(28.6)$ \\
TgAb reduction $>50 \%$ & $10(47.6)$ \\
Negative TgAb &
\end{tabular}

NIFTP, noninvasive follicular thyroid neoplasm with papillary-like nuclear features; $\mathrm{Tg}$, thyroglobulin; $\mathrm{TgAb}$, anti-thyroglobulin antibodies.

*With negative $\mathrm{Tg}$

Unfortunately, we did not evaluate this score in our cytology samples. Although we did not perform molecular tests, previous studies have shown that mutations in BRAF are not expected in NIFPT (Nikiforov et al. 2016), while mutations in RAS (Nikiforov et al. 2016) and a 'suspicious' profile in the Afirma Gene Expression Classifier test (Wong et al. 2016) may be observed.

The median follow-up time was longer than 5 years. In this respect, it is known that $80 \%$ of recurrences occur in these first years. None of the 129 patients presented structural disease or an increase in $\mathrm{Tg}$ or $\mathrm{TgAb}$ during follow-up, although they did not receive adjuvant therapy with radioiodine and half of them were submitted only to lobectomy. These results confirm the indolent course of these lesions after complete resection.

In conclusion, in this study, NIFTP corresponded to $15 \%$ of the cases diagnosed as PTC. The appearance of NIFTP as a highly suspicious nodule on US was uncommon (5\%). Malignant cytology was observed in only $4 \%$ of cases of NIFTP and cytology was indeterminate in most patients (62\%). Finally, none of the 129 patients with NIFTP (65 submitted to lobectomy and none of them receiving radioiodine) developed structural recurrence or had an increase in Tg or TgAb.

Declaration of interest

The authors declare that there is no conflict of interest that could be perceived as prejudicing the impartiality of the research reported.

\section{Funding}

This research did not receive any specific grant from any funding agency in the public, commercial or not-for-profit sector.

Published by Bioscientifica Ltc.
(C) 2016 Society for Endocrinology Printed in Great Britain 


\section{Author contribution statement}

G F Mourão and P W Rosario provided the study design. Study conduct was provided by G F Mourão and M B Nunes. Data management was done by G F Mourão, P W Rosario and M S Nunes. Data analysis was done by G F Mourão and P W Rosario. Data interpretation was done by G F Mourão, $\mathrm{P}$ W Rosario and M B Nunes. All authors contributed to the manuscript writing, review and approval.

\section{References}

Faquin WC, Wong LQ, Afrogheh AH, Ali SZ, Bishop JA, Bongiovanni M, Pusztaszeri MP, VandenBussche CJ, Gourmaud J, Vaickus LJ, et al. 2016 Impact of reclassifying noninvasive follicular variant of papillary thyroid carcinoma on the risk of malignancy in the Bethesda system for reporting thyroid cytopathology. Cancer Cytopathology 124 181-187. (doi:10.1002/cncy.21631)

Haugen BR, Alexander EK, Bible KC, Doherty GM, Mandel SJ, Nikiforov YE, Pacini F, Randolph GW, Sawka AM, Schlumberger M, et al. 2016 American Thyroid Association Management guidelines for adult patients with thyroid nodules and differentiated thyroid cancer: the American Thyroid Association guidelines task force on thyroid nodules and differentiated thyroid cancer. Thyroid 26 1-133. (doi:10.1089/thy.2015.0020)

Jang EK, Kim WG, Choi YM, Jeon MJ, Kwon H, Baek JH, Lee JH, Kim TY, Shong YK, Song DE, et al. 2015 Association between neck ultrasonographic findings and clinico-pathological features in the follicular variant of papillary thyroid carcinoma. Clinical Endocrinology 83 968-976. (doi:10.1111/cen.12674)

Maletta F, Massa F, Torregrossa L, Duregon E, Casadei GP, Basolo F, Tallini G, Volante M, Nikiforov YE \& Papotti M 2016 Cytological features of "noninvasive follicular thyroid neoplasm with papillary-like nuclear features" and their correlation with tumor histology. Human Pathology 54 134-142. (doi:10.1016/j. humpath.2016.03.014)

Mehrzad R, Nishino M, Connolly J, Wang H, Mowschenson P \& Hasselgren PO 2016 The relationship between the follicular variant of papillary thyroid cancer and follicular adenomas. Surgery 159 1396-1406. (doi:10.1016/j.surg.2015.11.026)

Nikiforov YE, Seethala RR, Tallini G, Baloch ZW, Basolo F, Thompson LD, Barletta JA, Wenig BM, Al Ghuzlan A, Kakudo K, et al. 2016 Nomenclature revision for encapsulated follicular variant of papillary thyroid carcinoma: a paradigm shift to reduce overtreatment of indolent tumors. JAMA Oncology 2 1023-1029. (doi:10.1001/jamaoncol.2016.0386)

Pradeep PV 2016 Ramifications of new terminology for encapsulated follicular variant of papillary thyroid carcinoma. JAMA Oncology 2 1097-1098. (doi:10.1001/jamaoncol.2016.2211)

Rosario PW, Penna GC \& Calsolari MR 2014 Noninvasive encapsulated follicular variant of papillary thyroid carcinoma: is lobectomy sufficient for tumours $\geq 1 \mathrm{~cm}$ ? Clinical Endocrinology 81 630-632. (doi:10.1111/cen.12387)

Strickland KC, Howitt BE, Marqusee E, Alexander EK, Cibas ES, Krane JF \& Barletta JA 2015 The impact of noninvasive follicular variant of papillary thyroid carcinoma on rates of malignancy for fine-needle aspiration diagnostic categories. Thyroid 25 987-992. (doi:10.1089/ thy.2014.0612)

Strickland KC, Vivero M, Jo VY, Lowe AC, Hollowell M, Qian X, Wieczorek T, French C, Teot L, Sadow PM, et al. 2016 Pre-operative cytologic diagnosis of noninvasive follicular thyroid neoplasm with papillary-like nuclear features (NIFTP): a prospective analysis. Thyroid 26 1466-1471. (doi:10.1089/thy.2016.0280)

Wong KS, Angell TE, Strickland KC, Alexander EK, Cibas ES, Krane JF \& Barletta JA 2016 Noninvasive follicular variant of papillary thyroid carcinoma and the Afirma gene-expression classifier. Thyroid $\mathbf{2 6}$ 911-915. (doi:10.1089/thy.2015.0644)

Received in final form 19 September 2016

Accepted 22 September 2016

Accepted Preprint published online 22 September 2016
(C) 2016 Society for Endocrinology Printed in Great Britain
Published by Bioscientifica Ltd 